\title{
Coherence between oscillations in the cardiorespiratory system and tissue oxygen index in muscle recovering from intensive exercise in humans
}

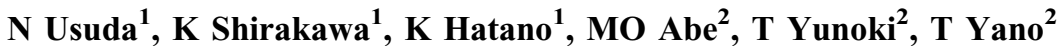 \\ ${ }^{1}$ Graduate school of Education, Hokkaido University, Sapporo, Japan \\ ${ }^{2}$ Department of Human Developmental Sciences, Faculty of Education, Hokkaido University, Sapporo, Japan
}

Received: April 1, 2019

Accepted: August 11, 2019

\begin{abstract}
It has been shown that the tissue oxygen index (TOI) measured by near-infrared spectroscopy oscillates at very low frequencies during recovery after exercise and that this oscillation is derived from interactions among biochemical substances involved in oxidative metabolism in skeletal muscle. As a further step, we examined whether TOI in muscle interacts through oscillation with factors related to oxygen in the cardiorespiratory system. For this examination, coherence and phase difference between the TOI in the vastus lateralis and heart rate (HR) and between TOI and arterial oxygen saturation $\left(\mathrm{SpO}_{2}\right)$ were sequentially determined during recovery $(2-60 \mathrm{~min})$ after severe cycle exercise with a workload of $7.5 \%$ of body weight for $20 \mathrm{~s}$. Significant coherence between TOI and HR was obtained in the very low-frequency band (approximate range: $0.002-0.03 \mathrm{~Hz}$ ) and in the low-frequency band (approximate range: $0.06-0.12 \mathrm{~Hz}$ ). The phase difference was negative in the low-frequency band and positive in the very low-frequency band. The coherence between TOI and $\mathrm{SpO}_{2}$ was significant in the very low-frequency band. The phase difference was negative. There were no sequential changes in these coherences and phase differences. The results suggest that TOI in skeletal muscle interrelates with factors related to the heart and lungs.
\end{abstract}

Keywords: coherence, cardiorespiratory system, tissue oxygen index, oscillation, recovery from exercise

\section{Introduction}

Near-infrared spectroscopy (NIRS) is a method for non-invasively measuring tissue oxygen index (TOI). It has been reported that intramuscular TOI measured by this method oscillates during recovery from exercise (22). In that study, the power spectral density (PSD) of intramuscular TOI after exercise was calculated using fast Fourier transformation. The maximum PSD of TOI appeared in the very low-frequency band $(0.0039-0.0061 \mathrm{~Hz})(22)$. Moreover, from evidence $(7,8)$ indicating that the process of resynthesis of creatine phosphate accompanying oxidative phosphorylation oscillated in the very low-frequency band $(0.002-0.025 \mathrm{~Hz})$ after exercise, it was suggested that TOI oscillates due to oxidative metabolism (22). Furthermore, it was pointed out by referring to Richard's oscillation model (15) that the oscillation of TOI was caused by feedback of adenosine triphosphate (ATP) to oxidative phosphorylation (22). Recently, it has been reported that TOI oscillates in

\footnotetext{
Corresponding author: Noboru Usuda

Department of Human Development Sciences, Faculty of Education, Hokkaido University

Kita-11, Nishi-7, Kita-ku, Sapporo 060-0811, Japan

E-mail: n-usuda@g.ecc.u-tokyo.ac.jp

Current address: Noboru Usuda

Department of Life Sciences, Graduate school of Arts and Sciences, The University of Tokyo

Komaba, 3-8-1, Meguro-ku, Tokyo 153-8902, Japan
} 
the very low-frequency band $(0.0039-0.0048 \mathrm{~Hz})$ during exercise $(21)$. In that report, in addition to the feedback process of ATP, it was pointed out that the interactions among biochemical substances of the tricarboxylic acid (TCA) cycle cause oscillation of NADH and that TOI oscillates, since NADH is involved in oxidative phosphorylation in the electron transport system (21). In fact, it has been observed that NADH oscillated when the TCA cycle was activated (1), although that observation was in an animal experiment. Thus, it has been shown that oscillation of TOI is derived mainly from oscillation related to oxygen metabolism in muscle fibers in the very-low-frequency band.

Since oscillation has the property of entrainment, if there is oscillation in each organ, it is possible for the oscillation to be propagated by entrainment. For example, the entrainment between muscle contraction rhythm and respiration rhythm has been studied for a long time $(2,14,16)$. In addition, it is known that muscle pump action accompanying muscle contraction during posture control affects circulation function (17). In other words, there seems to be an entrainment between muscle contraction and cardiorespiratory function. However, as far as we know, entrainment between the oscillation of oxidative metabolism in muscle and the oscillation of cardiorespiratory function has not been studied.

Meanwhile, intramuscular TOI reflects the balance between oxygen supply and consumption $(3,9)$. Therefore, there is also the possibility that the oscillation of oxygen supply affects the oscillation of TOI in muscle. Oxygen supply to active muscle depends on the product of cardiac output and arterial oxygen content [i.e., heart rate (HR), stroke volume, arterial oxygen saturation, and arterial oxygen partial pressure]. Therefore, these indicators are factors of the rhythm phenomenon of TOI in muscle. In addition to this, carbon dioxide $\left(\mathrm{CO}_{2}\right)$ produced by oxidative metabolism has the property of vasodilatation (12). Therefore, it can be deduced that end-tidal $\mathrm{CO}_{2}$ partial pressure $\left(\mathrm{PETCO}_{2}\right)$ can cause TOI oscillation (23).

Thus, the oscillation of TOI is not a simple phenomenon derived from interactions among biochemical substances related to oxidative metabolism in skeletal muscle. This oscillation may possibly have a function of correlating with each organ of the respiratory and circulatory systems outside skeletal muscle due to entrainment. Therefore, in this study, we examined the interaction between the oscillation of TOI and the oscillation of factors related to oxygen in the heart and lungs.

\section{Materials and Methods}

Subjects

The subjects were eight healthy men (age: $23 \pm 2.4$ years, height: $170.7 \pm 8.1 \mathrm{~cm}$, weight: $64.5 \pm 10.3 \mathrm{~kg}$ ). Each subject signed a statement of informed consent following a full explanation regarding the nature of the experiment.

\section{Study procedures}

In this study, each subject performed an intensive exercise for $20 \mathrm{~s}$ on a cycle ergometer (PowermaxVII, COMBI, Tokyo, Japan) with a workload of $7.5 \%$ of body weight. Before the exercise, each subject rested on the cycle ergometer for $10 \mathrm{~min}$. After the exercise, each subject got off the cycle ergometer and moved to a chair to decrease body motion and recovered for $60 \mathrm{~min}$. 


\section{Measurement}

Blood samples (100 $\mu \mathrm{l}$ each) were collected from fingers using a capillary tube. Each subject's hand was prewarmed in $40-45^{\circ} \mathrm{C}$ water while sitting on the ergometer to arterialize capillary blood (25). After this warming, the subject's hand was warmed by a heating glove at rest, during exercise and during recovery. It has been shown that such blood samples might not accurately reflect arterial $\mathrm{O}_{2}$ pressure but can closely reflect arterial $\mathrm{pH}$. Samples were analyzed using a blood gas analyzer (i-STAT, I-Stat, Abbon Point of Care Inc., IL, USA) to measure $\mathrm{pH}$ and lactate.

TOI was measured in the vastus lateralis by NIRS (NIRO200x, Hamamatsu Photonics, K.K. Hamamatsu, Japan) with a sampling rate of $1 \mathrm{~Hz}$. The NIRS system consisted of irradiation and detecting devices, and the distance between the devices was $40 \mathrm{~mm}$. Three different near-infrared rays $(735,810$, and $850 \mathrm{~nm})$ from the irradiation devices penetrated into muscle, where they were scattered or absorbed in tissues and transmitted to the detecting device. In this study, we used spatially resolved spectroscopy (SRS). TOI was calculated by the ratio of oxy-hemoglobin $\left(\mathrm{O}_{2} \mathrm{Hb}\right)$ and total hemoglobin $(\mathrm{Hb})$ using the following formula:

$$
\mathrm{TOI}=\frac{\mathrm{O}_{2} \mathrm{Hb}}{\text { Total } \mathrm{Hb}} \times 100(\% \mathrm{TOI})
$$

We measured arterial oxygen saturation $\left(\mathrm{SpO}_{2}\right)$ from the tip of a finger of the left hand using an oxygen saturation degree monitoring apparatus (PULSOX-300i, KONICA MINOLTA, Tokyo, Japan). The rates of absorption of red-color rays and infrared rays are different, and the oxygen saturation degree monitoring apparatus uses the differences to estimate $\mathrm{SpO}_{2}$.

$\mathrm{PETCO}_{2}$ was measured by a respiratory gas analyzer (AEROMONITOR AE-310S, Minato Medical Science CO. Ltd., Osaka, Japan) in the breath-by-breath mode. Expiration and inspiration flow rates were measured by a heat wire flowmeter. A 2.01 syringe was used to calibrate the flowmeter. The $\mathrm{CO}_{2}$ fraction was measured by an infrared absorption analyzer, and the $\mathrm{O}_{2}$ fraction was measured by a magnetic dumbbell analyzer. To calibrate these sensors, we used standard density gas $\left(\mathrm{O}_{2}: 15.38 \%\right.$, $\mathrm{CO}_{2}: 5.132 \%$ ). HR was measured by a $\mathrm{HR}$ monitor connected to the respiratory gas analyzer.

\section{Data analysis}

$\mathrm{HR}$ and $\mathrm{PETCO}_{2}$ breath-by-breath data were converted to $1 \mathrm{~s}$ data by the interpolation method.

Data obtained from 2 to 60 min during recovery were used for analysis. Data for the first 2 min after exercise were not used for analysis, because subjects utilized that time for getting off the cycle ergometer.

All signals were filtered using a band bass $(0.001-0.2 \mathrm{~Hz})$ to focus on the low-frequency oscillation, which is shown to associate with oxygen metabolism in preceding studies $(7,8,22)$. Data for analysis consisted of oscillation around the axis and level of the axis of oscillation. Trend of the level of axis was removed using the detrend function in Matlab software (Mathworks Inc., Natick, MA, USA). 
Coherence analysis and phase analysis

Coherence analysis was carried out to determine the common frequency characteristics between two signals over time between 2 and 60 min during the recovery phase. In this study, the coherence between TOI and other items $\left(\mathrm{HR}, \mathrm{SpO}_{2}\right.$, and $\left.\mathrm{PETCO}_{2}\right)$ was calculated. Data of 1,500 points were taken as the basis of the discrete Fourier transform (DFT). We split this DFT into three parts with 512 samples of a Blackman window. In addition, while maintaining 1,500 points, by sequentially moving 100 DFT points along the recovery time axis, time series DFT (Rxy) during recovery was obtained.

$$
\left|R_{x y}(\lambda)\right|^{2}=\frac{\left|f_{x y}(\lambda)\right|^{2}}{f_{x x}(\lambda) f_{y y}(\lambda)},
$$

where $\lambda$ is frequency, $f_{x x}(\lambda)$ and $f_{y y}(\lambda)$ represent each signal, and $f_{x y}$ is a cross spectrum. The phase difference was calculated by the cross spectrum.

\section{Statistical analyses}

The results are presented as means \pm standard deviations. Levels of blood $\mathrm{pH}$ and blood lactate significantly different from resting values were tested by Dunnett's method. The significance level was set at $p<0.05$. The following numerical expression was used to determine the significance level $(p<0.05)$ of coherence and phase difference:

$$
\left|R_{x y}(\lambda)\right|_{1-a}^{2}=1-a^{1 /(N e-1)}
$$

where $\mathrm{Ne}$ represents the degrees of equivalent freedom and $a$ represents the statistically significant level.

\section{Ethics}

The Ethics Committee of Hokkaido University Graduate School of Education approved this study. This study was performed in accordance with the Declaration of Helsinki.

\section{Results}

The maximum power with the cycle ergometer was $671.9 \pm 11.6 \mathrm{~W}$. Table I shows blood $\mathrm{pH}$ and lactate at rest and during recovery. Blood lactate significantly increased after exercise and then decreased toward the resting level. Blood $\mathrm{pH}$ was also significantly decreased after exercise. The blood $\mathrm{pH}$ recovered to the resting value at post $60 \mathrm{~min}$ from exercise.

Table I. Mean value and standard deviation of arterial blood $\mathrm{pH}$ and blood lactate at pre-exercise and post-exercise

\begin{tabular}{|l|c|c|c|c|c|}
\hline & Pre & Post 5 min & Post 15 min & Post 30 min & Post 60 min \\
\hline $\mathrm{pH}$ & $7.39 \pm 0.02$ & $7.21 \pm 0.03^{\dagger}$ & $7.26 \pm 0.03^{\dagger}$ & $7.32 \pm 0.03^{\dagger}$ & $7.31 \pm 0.02$ \\
\hline $\mathrm{Lac}^{-}(\mathrm{mmol} / \mathrm{L})$ & $1.09 \pm 0.24$ & $12.77 \pm 1.45^{\dagger}$ & $11.63 \pm 1.42^{\dagger}$ & $7.62 \pm 1.24^{\dagger}$ & $3.01 \pm 0.38^{\dagger}$ \\
\hline
\end{tabular}

${ }^{\dagger}$ Significant differences from pre-exercise $(p<0.05)$ 
Figure 1 shows typical data for TOI, $\mathrm{HR}, \mathrm{SpO}_{2}$, and $\mathrm{PETCO}_{2}$ during recovery from exercise. TOI rapidly decreased approximately $10 \mathrm{~s}$ after the start of exercise and increased rapidly immediately after stopping the exercise. Then, TOI recovered to the resting value and oscillated. HR increased after the start of exercise and rapidly decreased during recovery. During the recovery phase, $\mathrm{HR}$ oscillated. $\mathrm{SpO}_{2}$ decreased slightly in the exercise phase. In the recovery phase, $\mathrm{SpO}_{2}$ recovered to the resting value and then oscillated at that time. $\mathrm{PETCO}_{2}$ was maintained at the resting value in half of the subjects and decreased rapidly after the start of exercise in half of the subjects. Then, after rapidly increasing, $\mathrm{PETCO}_{2}$ decreased to the resting value and then oscillated at that time.

The following results were obtained by analysis of data obtained between 2 and $60 \mathrm{~min}$ during the recovery period.

Figure 2 shows the results for TOI-HR coherence. TOI-HR coherence was statistically significant between the lower limit of about $0.06 \mathrm{~Hz}$ and the upper limit of about $0.12 \mathrm{~Hz}$ (low-frequency band), and the phase difference of the band was negative. TOI-HR coherence was also statistically significant between the lower limit of about $0.002 \mathrm{~Hz}$ and the upper limit of about $0.03 \mathrm{~Hz}$ (very low-frequency band). The phase difference of the band was positive. Figure 3 shows the results for TOI-SpO $\mathrm{S}_{2}$ coherence. There was significant coherence between the lower limit of about $0.002 \mathrm{~Hz}$ and the upper limit of about $0.03 \mathrm{~Hz}$. The phase difference of the band was negative. Figure 4 shows the results for TOI-PETCO ${ }_{2}$ coherence. There was significant coherence at various frequencies, but it was scattered. The phase difference of the band was not clear due to the scattered results.

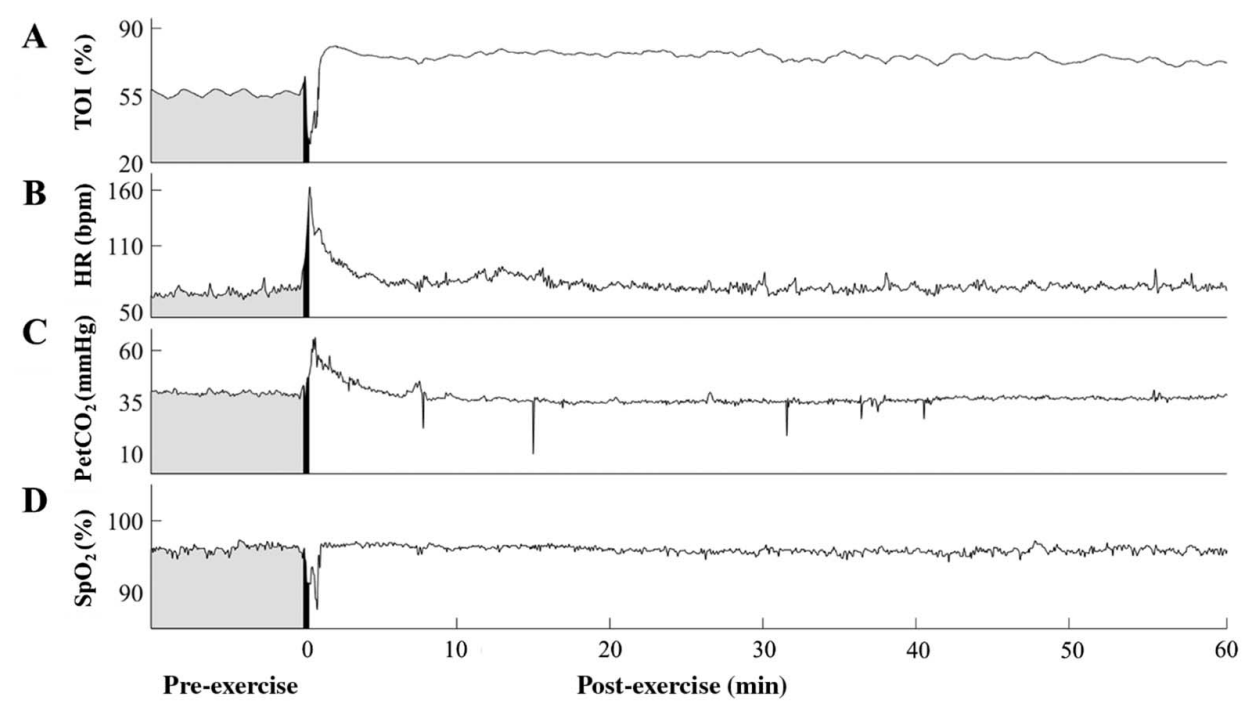

Fig. 1. Data for one subject. Raw data of (A) TOI in the vastus lateralis, (B) $\mathrm{HR},(\mathrm{C}) \mathrm{SpO}_{2}$, and (D) $\mathrm{PETCO}_{2}$ for entire time (rest: $10 \mathrm{~min}$, exercise: $20 \mathrm{~s}$, recovery: $60 \mathrm{~min}$ ). The gray area represents rest, the black area represents exercise, and the white area represents recovery 

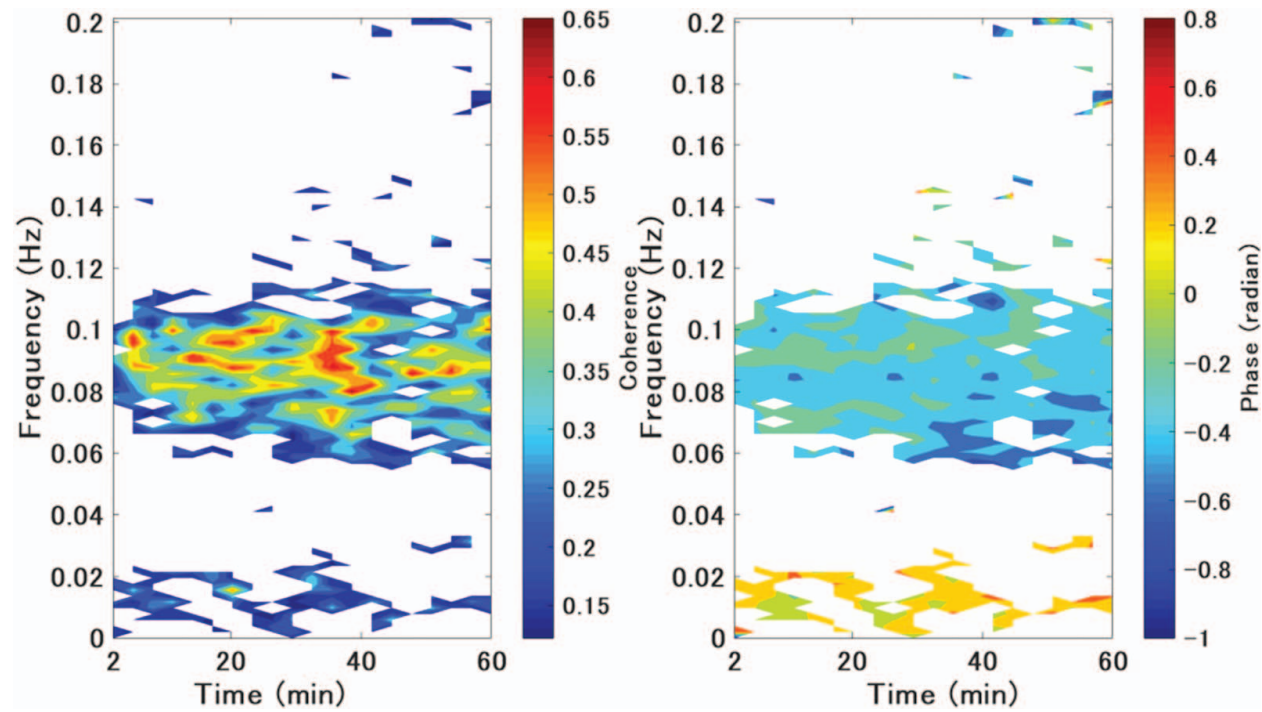

Fig. 2. Pooled data from eight subjects during recovery (2-60 min). (A) Pooled TOI-HR coherence and (B) pooled TOI-HR phase difference are shown. The coherence scale is represented in a color scale (the highest is red, the lowest is blue, and no significant coherence is white). The phase scale is represented in a color scale (no phase difference is zero, the positive highest phase difference is red, and the negative highest difference is blue)
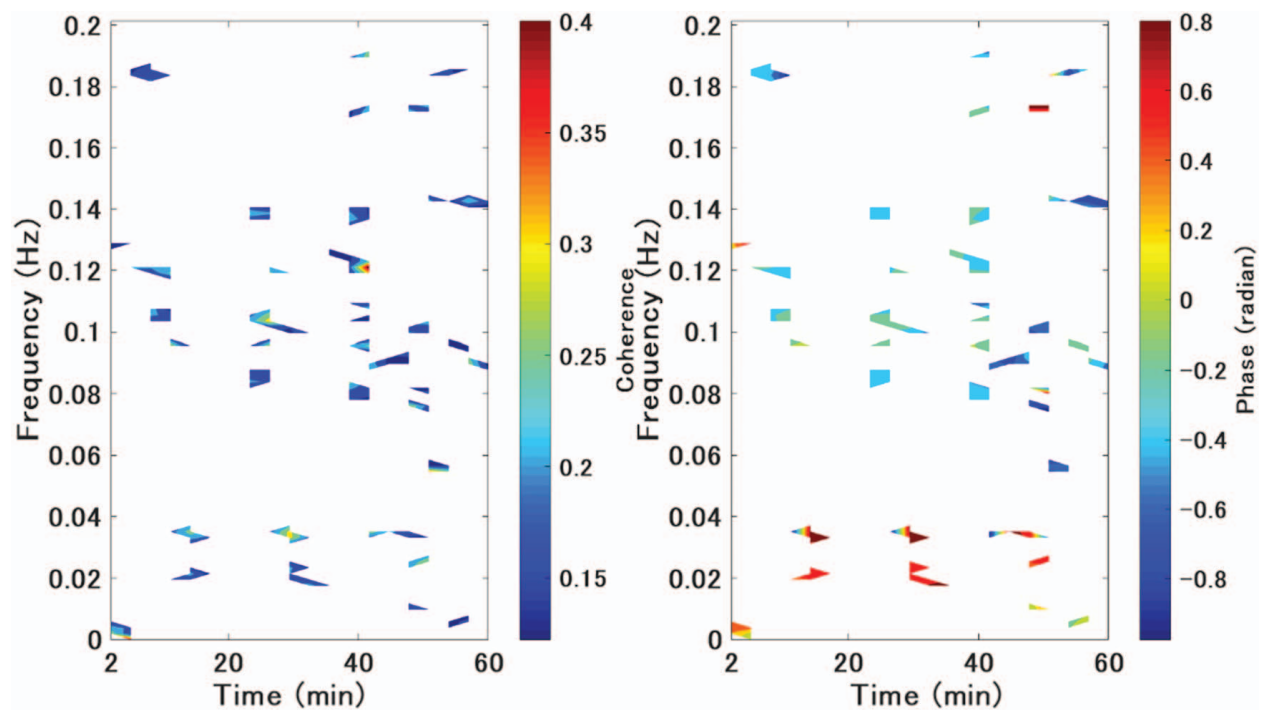

Fig. 3. Pooled data from eight subjects during recovery $(2-60 \mathrm{~min})$. Pooled TOI-PETCO ${ }_{2}$ coherence and pooled TOI-PETCO 2 phase difference are shown. The coherence scale is represented in a color scale (the highest is red, the lowest is blue, and no significant coherence is white). The phase scale is represented in a color scale (no phase difference is zero, the positive highest phase difference is red, and the negative highest difference is blue) 

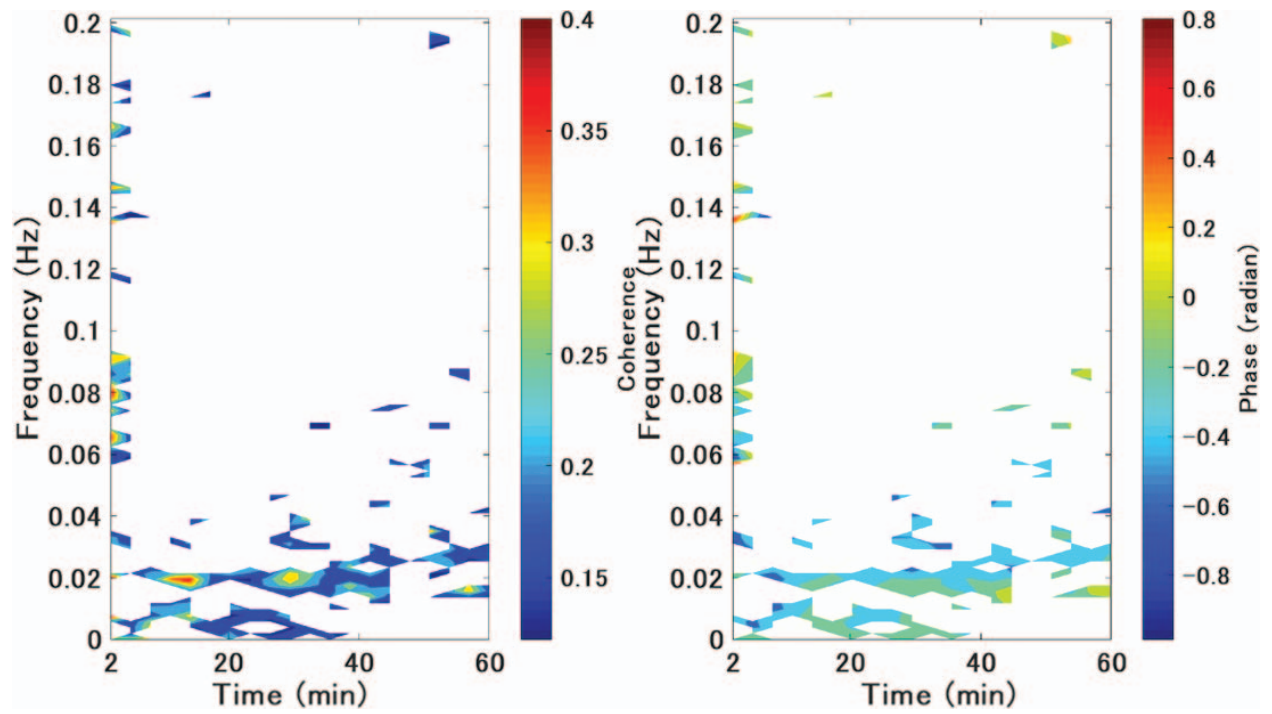

Fig. 4. Pooled data from eight subjects during recovery $(2-60 \mathrm{~min})$. Pooled TOI-SpO $\mathrm{S}_{2}$ coherence and pooled TOI- $\mathrm{SpO}_{2}$ phase difference are shown. The coherence scale is represented in a color scale (the highest is red, the lowest is blue, and no significant coherence is white). The phase scale is represented in a color scale (no phase difference is zero, the positive highest phase difference is red, and the negative highest difference is blue)

\section{Discussion}

TOI oscillation affected by dual factors

NIRS is a non-invasive way to measure muscle blood flow and oxygenation (4). It has been shown that muscle oxygenation measured by NIRS using the Beer-Lambert method (BLM) oscillates (24). However, oxygenation measured by the BLM is affected by skin blood flow (11). Skin blood flow has very low-frequency components (10). Therefore, oscillation of TOI determined by SRS, which is not affected by skin blood flow rate, has been confirmed in the very low-frequency band $(21,22)$.

TOI in skeletal muscle reflects the balance between oxygen supply and oxygen consumption $(3,9)$. For example, a decrease in the level of TOI (decline in the axis of oscillation) is observed after the start of exercise. This is because oxygen consumption exceeds oxygen supply. On the other hand, immediately after the exercise, it is possible to observe an overshoot of the axial level of TOI in skeletal muscle (elevation of the axis of oscillation) because oxygen supply exceeds oxygen consumption. However, these explanations are not applicable to the rising and falling phases observed during the oscillation of TOI. This can be understood by the following simple model (20). First, TOI is expressed by the difference between oxygen supply and oxygen consumption. Next, the oscillation of oxygen supply is represented by a sine curve. Similarly, the oscillation of oxygen consumption is represented by a sine curve having a phase difference from the oxygen supply. Then, the oscillation of TOI, which is the difference between them, becomes a sine curve. When the level of each axis of oscillations of oxygen consumption and oxygen supply is changed, the difference between the two axis levels appears as a change in the axis level of TOI. Since the level of the axis of TOI decreases during exercise, it can be deduced that the change in the level of oxygen consumption is greater than the level of oxygen supply. Even in this case, 
there is oscillation of TOI, although it is difficult to observe in a short exercise such as that this study. From this point of view, the overall image of the oscillation of TOI should be reviewed from the relationship between oxygen supply and consumption.

\section{Influence of cardiorespiratory system on TOI (from $\mathrm{HR}, \mathrm{SpO}_{2}$, and $\mathrm{PETCO}_{2}$ )}

Oxygen supply to active muscles is determined by cardiac output and arterial oxygen content. Cardiac output is the product of stroke volume and HR, and arterial oxygen content is the sum of Hb-bound oxygen and the amount of oxygen dissolved in the blood. Arterial oxygen content reflects gas exchange in the lungs.

The PSD of human HR fluctuation has been found to have components in the lowfrequency band (less than $0.12 \mathrm{~Hz}$ ) and in the high-frequency band $(0.224-0.28 \mathrm{~Hz})(20)$. The high-frequency band is mediated by the parasympathetic nervous system. However, this frequency band was not examined in this study, and we therefore cannot discuss it. Moreover, low-frequency components have not been observed for oxidative metabolism related to the recovery phase of TOI or creatine phosphate. Therefore, it is likely that the coherence in the low-frequency band found in this study indicates the existence of propagation from the heart to TOI of skeletal muscle through oscillation of cardiac output.

There was significant coherence between TOI and $\mathrm{SpO}_{2}$ in the very low-frequency band. Although $\mathrm{SpO}_{2}$ is a value related to arterial oxygen content and a value determined in the lung, owing to measuring the value at the fingertip, there is a time lag from oxygen saturation in the lung. Therefore, the time difference between $\mathrm{SpO}_{2}$ determined at the fingertip and TOI determined in the lateral vastus muscle corresponds to the delay time. However, since $\mathrm{SpO}_{2}$ reflects the value of arterial blood, this item should be involved in oxygen supply. Therefore, it is believed that propagation of $\mathrm{SpO}_{2}$ oscillation from the lungs to skeletal muscle exists in the very low-frequency band.

The effect of $\mathrm{CO}_{2}$ accumulation by artificially manipulating respiratory resistance on oxygenation of the brain and active muscles has been investigated (12). As a result, it has been observed that oxygenation rose at both sites as $\mathrm{PETCO}_{2}$ increased. It was also suggested that these increases in oxygenation were derived from an increase in blood flow. However, this effect is derived from the level of $\mathrm{CO}_{2}$ and not from the $\mathrm{CO}_{2}$ oscillation. Therefore, we cannot directly link this result with the results of this study.

Yano et al. (23) proposed from evidence of cross correlations of pulmonary ventilation, rather, and $\mathrm{PETCO}_{2}$ that the dynamic homeostasis of $\mathrm{PETCO}_{2}$ is maintained as follows: (1) there is a feedback signal via the carotid artery of $\mathrm{PETCO}_{2}$, (2) $\mathrm{CO}_{2}$ is excreted by ventilation, and (3) $\mathrm{CO}_{2}$ is transported to the lung due to cardiac output. In addition, the result of these interactions seems to induce $\mathrm{PETCO}_{2}$ oscillation. Furthermore, it was inferred that oscillation of $\mathrm{PETCO}_{2}$ produced oscillation of oxygenation. However, the results of this study suggest that the oscillation of $\mathrm{PETCO}_{2}$ has little effect on the oscillation of intramuscular TOI.

\section{Influence of TOI on heart}

We stated in the introduction that (1) there was the possibility that TOI oscillated in relation to oxidative phosphorylation of the electron transfer system, that (2) the recovery process of creatine phosphate after exercise oscillated and its recovery was due to oxidative phosphorylation, and that (3) the oscillation of TOI after exercise was related to oxygen consumption. Moreover, it was an important point that the oscillations described in (2) and (3) occurred in very low-frequency bands. This suggests that TOI of the very low-frequency band is related 
to oxygen consumption in skeletal muscle fibers $(21,22)$. Therefore, oxygen consumption probably oscillates within skeletal muscle fibers. As a further step in this study, it was shown that the TOI oscillation is propagated to the heart.

In physiology, we are interested in the mechanism by which the relationship between TOI and HR is established. In this study, it is physiologically significant that TOI oscillation propagated to HR oscillation. Since we examined the results obtained after exercise, the signal output from muscle mechanical receptors (19) or central command (5) would have had little effect on HR. The muscle metaboreflex mainly affects HR [see references $(6,13,18,23)$ for muscle metaboreflex, although oscillations of metabolites are not described in those reports]. In this study, there are some points to note about metaboreflex. The oscillation was distinguished from the axial level of oscillation and the influence of this axial level was removed in this study. As a result, in the recovery period after the exercise, the TOI oscillation had coherence with the HR oscillation, despite a change in the level of the muscle metabolism. Therefore, this coherence is basically an entrainment between them. In addition, the relationship between muscle metaboreflex and HR level does not seem to be completely clear unless consideration is given to changes in the level of TOI. However, the entrainment at a very low-frequency found in this study is a new finding that will be valuable for examining the mechanism of muscle metaboreflex.

\section{Interaction in the respiratory circulatory systems (centering on TOI)}

It is possible to reexamine the relationship between each of the physiological items described above from the phase difference. However, it should be noted that the phase difference does not always represent a causal relationship. For example, when the phase difference is $90^{\circ}$, there may be a phase difference of $-270^{\circ}$. Although there are such restrictions, it is one of the useful tools for examining the causal relationship.

In the very low-frequency band, the phase difference between TOI and HR was negative. This means that TOI precedes HR. Therefore, it can be interpreted from the phase difference that HR influences TOI in the low-frequency band. Furthermore, since the phase difference of $\mathrm{SpO}_{2}$ was negative, it can be concluded that $\mathrm{SpO}_{2}$ affects TOI. These relationships are consistent with what has been discussed above.

As mentioned above, TOI influenced HR. Simultaneously, the oxygen supply system ( $\mathrm{HR}$ and $\mathrm{SpO}_{2}$ ) affected TOI. HR is a function of the heart pumping and $\mathrm{SpO}_{2}$ is an element determined in the lungs. In addition, it is believed that the oscillation of TOI itself is caused by the interactions among biochemical substances of the oxidative metabolism in muscles and by the oscillation of oxygen supply. The oscillation of this TOI seemed to have interactions with the oscillation of elements of the respiratory circulation system. These interactions may involve order formation in the cardiorespiratory system, but further examination will be necessary to elucidate this point.

\section{Conclusions}

The coherence of TOI and HR and that of TOI and $\mathrm{SpO}_{2}$ did not change with time regardless of the metabolic change after exercise. The oscillation of each item should be distinguished from the magnitude of axial oscillation. An influence of oscillation related to oxygen supply on TOI oscillation was found in the low-frequency band (HR-TOI) and the very low-frequency band (TOI-SpO$)_{2}$ ). Oscillation of TOI related to oxygen consumption in 
skeletal muscle affected HR in the very low-frequency band. These results suggest that TOI in skeletal muscle is interrelated to the factors related to the heart and lungs.

\section{REFERENCES}

1. Aon MA, Cortassa S, Marbán E, O’Rourke B: Synchronized whole cell oscillations in mitochondrial metabolism triggered by a local release of reactive oxygen species in cardiac myocytes. J. Biol. Chem. 278, 44735-44744 (2003)

2. Bechbache RR, Duffin J: The entrainment of breathing frequency by exercise rhythm. J. Physiol. 272, 553-561 (1977)

3. Boushel R, Langberg H, Olesen J, Gonzales-Alonzo J, Bülow J, Kjaer M: Monitoring tissue oxygen availability with near infrared spectroscopy (NIRS) in health and disease. Scand. J. Med. Sci. Sports. 11, 213-222 (2001)

4. Chance B, Nioka S, Kent J, McCully K, Fountain M, Greenfeld R, Holtom G: Time-resolved spectroscopy of hemoglobin and myoglobin in resting and ischemic muscle. Anal. Biochem. 174, 698-707 (1988)

5. Dampney RA: Central neural control of the cardiovascular system: current perspectives. Adv. Physiol. Educ. 40, 283-296 (2016)

6. Fisher JP, Adlan AM, Shantsila A, Secher JF, Sørensen H, Secher NH: Muscle metaboreflex and autonomic regulation of heart rate in humans. J. Physiol. 591, 3777-3788 (2013)

7. Iotti S, Borsari M, Bendahan D: Oscillations in energy metabolism. Biochim. Biophys. Acta. 1797, 1353-1361 (2010)

8. Iotti S, Gottardi G, Clementi V, Barbiroli B: The mono-exponential pattern of phosphocreatine recovery after muscle exercise is a particular case of a more complex behaviour. Biochim. Biophys. Acta. 1608, 131-139 (2004)

9. Koga S, Barstow T J, Okushima D, Rossiter H B, Kondo N, Ohmae E, Poole DC: Validation of a high-power, time-resolved, near-infrared spectroscopy system for measurement of superficial and deep muscle deoxygenation during exercise. J. Appl. Physiol. (1985) 118, 1435-1442 (2015)

10. Kvernmo H, Stefanovska A, Kirkenboen K, Kvernebo K: Oscillations in the human cutaneous blood perfusion signal modified by endothelium-dependent and endothelium-independent vasodilators. Microvasc. Res. 57, 298-309 (1999)

11. Messere A, Roatta S: Influence of cutaneous and muscular circulation on spatially resolved versus standard Beer-Lambert near-infrared spectroscopy. Physiol. Rep. 1, e00179 (2013)

12. Nielsen HB, Boesen M, Secher NH: Near-infrared spectroscopy determined brain and muscle oxygenation during exercise with normal and resistive breathing. Acta Physiol. Scand. 171, 63-70 (2001)

13. Nishiyasu T, Tan N, Morimoto K, Nishiyasu M, Yamaguchi Y, Murakami N: Enhancement of parasympathetic cardiac activity during activation of muscle metaboreflex in humans. J. Appl. Physiol. (1985) 77, 2778-2783 (1994)

14. Perségol L, Jordan M, Viala D: Evidence for the entrainment of breathing by locomotor pattern in human. J. Physiol. (Paris) 85, 38-43 (1991)

15. Richard P: The rhythm of yeast. FEMS Microbiol. Rev. 27, 547-557 (2003)

16. Tiller NB, Price MJ, Campbell IG, Romer LM: Effect of cadence on locomotor-respiratory coupling during upper-body exercise. Eur. J. Appl. Physiol. 117, 279-287 (2017)

17. Verma AK, Garg A, Xu D, Bruner M, Fazel-Rezai R, Blaber AP, Tavakolian K: Skeletal muscle pump drives control of cardiovascular and postural systems. Sci. Rep. 7, 45301 (2017)

18. Watanabe K, Ichinose M, Fujii N, Matsumoto M, Nishiyasu T: Individual differences in the heart rate response to activation of the muscle metaboreflex in humans. Am.J. Physiol. Heart. Circ. Physiol. 299, H1708-H1714 (2010)

19. Watanabe N, Hotta $\mathrm{H}$ : Heart rate changes in response to mechanical pressure stimulation of skeletal muscles are mediated by cardiac sympathetic nerve activity. Front. Neurosci. 10, 614 (2017)

20. Yano T, Afroundeh R, Arimitsu T, Yunoki T (in press): Relationship between tissue oxygen index in skeletal muscle and oxygen uptake at pulmonary level. Bulletin of Faculty of Education, Hokkaido University, Vol. 135

21. Yano T, Afroundeh R, Arimitsu T, Yunoki T (in press): Difference Between Low and High Intensity Exercises in the Amplitude of Oscillation of Tissue Oxygen Index. Bulletin of Faculty of Education, Hokkaido University, Vol. 135 
22. Yano T, Afroundeh R, Shirakawa K, Lian C, Shibata K: Oscillation in tissue oxygen index during recovery from exercise. Physiol. Res. 65, 259-269 (2016)

23. Yano T, Afroundeh R, Yamanaka R, Arimitsu T, Lian C, Shirkawa K, Yunoki T: Response of end tidal $\mathrm{CO}_{2}$ pressure to impulse exercise. Acta Physiol. Hung. 101, 103-111 (2014)

24. Yano T, Lian C, Arimitsu T, Yamanaka R, Afroundeh R, Shirakawa K, Yunoki T: Oscillation of oxygenation in skeletal muscle at rest and in light exercise. Acta Physiol. Hung. 100, 312-320 (2013)

25. Zavorsky GS, Cao J, Mayo NE, Gabbay R, Murias JM: Arterial versus capillary blood gases: a meta-analysis. Respir. Physiol. Neurobiol. 155, 268-279 (2007) 